\title{
Le tutorat : accompagnement de l'actualisation du dispositif
}

\author{
Didier Paquelin
}

Université Michel de Montaigne, Bordeaux3

Domaine Universitaire F-33607 Pessac cedex

paquelin@u-bordeaux3.fr

\begin{abstract}
RÉSUMÉ. L'apprentissage dans un dispositif de formation ouverte et à distance sollicite l'apprenant dans un processus singulier d'actualisation des fonctionnalités proposées par les concepteurs. Par une démarche intentionnelle, l'apprenant seul et accompagné construit une forme spécifique du dispositif qui différencie un état prescrit d'un état vécu. Cette appropriation intentionnelle et située est retenue comme principe directeur de cet article. A partir de résultats d'observations, l'auteur discute l'hypothèse de l'existence d'une zone proximale qui organise l'actualisation du dispositif prescrit. La dynamique ternaire de l'appropriation distingue différentes étapes complémentaires dont le déroulement suppose l'édification cognitive, affective et symbolique d'un cadre conteneur.

ABSTRACT. In order to learn in a distance open learning environment, the learner is required to bring into play specific processes that will actualize the functions offered by the designers. Relying on intentional procedures, the learner, alone and accompanied, builds up his or her own form of the system which enacts the difference between what was prescribed and what is actualized. That way of making the system his/her own intentionnally and according to the context is the guiding principle of this article. The author relies on the results of various observations and discusses the following hypothesis: there exists a proximal zone that organizes the actualization of the prescribed set up. $>$ From a threefold dynamic of appropriation several complementary phases can be distinguished. Their unfolding implies the cognitive, affective and symbolic build up of an enfolfding framework.
\end{abstract}

MOTS-CLÉS: accompagnement, appropriation, autonomie, coconstruction, dispositif de formation ouverte et à distance, interaction, genèse instrumentale, régulation.

KEYWORDS: accompaniment, autonomy, empowerment, coconstruction, interaction, open distance learning, regulation.

D\&S - 2-3/2004. Enigmes de la relation pédagogique, pages 157 à 182 


\section{Introduction}

Dans le contexte d'industrialisation de la formation, cet article questionnera la possible rationalisation du tutorat dans les dispositifs de formation ouverte et à distance en montrant les limites de la modélisation de l'acte éducatif, rappelant la nécessité de l'acceptation de l'indétermination (Aristote), voire de l'incertitude (Morin). Ce questionnement ne cherche pas à définir des propositions opérationnalisables, mais revient sur quelques fondamentaux des conditions d'apprentissage structurantes d'une démarche d'appropriation d'un dispositif par les acteurs (apprenants, tuteurs). Avant tout, il convient de préciser la posture épistémologique, sociale et humaine de l'auteur, qui considère l'apprenant comme un sujet acteur social, et en cela participant activement à la réalisation de ses apprentissages. Le propos de cet article trouve sa genèse dans le champ des théories de l'activité, de l'ergonomie du travail, de la sémiologie et de la psychopédagogie. Nous considérerons que l'apprentissage est un acte conscient et de conscientisation d'une transformation d'un état du soi, allant d'une transformation de sa base de connaissances, à la possible évolution ou transformation de ses valeurs, éthique et identité. Nous proposons plus spécifiquement d'aborder le tutorat non pas uniquement dans sa dimension relationnelle d'un sujet à un autre sujet, mais dans une dimension plus large qui est celle de l'implication participante de l'apprenant au processus de formation.

Pineau (2000) définit la formation comme «un processus unificateur de mise ensemble, en sens, d'éléments et moments autrement séparés». Cet auteur nous rappelle que le mot « former» est en équivalence avec « créer, constituer, composer, concevoir » mentionnant explicitement que l'être et la forme sont indissociables. Il rappelle l'inachèvement de cette forme dont l'évolution requiert implication et action du sujet se formant. La formation peut ainsi être reconnue comme rupture et reconstruction de forme. Se former c'est se mettre en mouvement, accepter son propre inachèvement, c'est chercher et entreprendre un cheminement vers la compréhension de soi, et de sa relation aux autres. Nous retrouvons cette dimension créatrice de la formation dans la définition que donne Linard (1998) d'un dispositif de formation: "fondé sur la mise en système des agents et des conditions d'une action... un dispositif est une construction cognitive fonctionnelle, pratique, incarnée. Il présuppose quelqu'un derrière la représentation préalable de l'effet visé et une logique de type dramatique qui combine la mise en scène des protagonistes, des rôles et des circonstances avec les règles du déroulement de l'action ». Si l'autonomie est fréquemment mentionnée comme requise à l'apprentissage dans des dispositifs de formation ouverte et à distance, le propos sera explicitement centré vers le processus d'appropriation d'un dispositif de formation ouverte et à distance. Nous chercherons à montrer comment et dans quelles conditions l'apprenant est susceptible d'exercer et de développer son autonomie dans le processus d'actualisation des fonctionnalités du dispositif. 
Le tutorat peut dès lors être analysé et interprété dans ce contexte. L'objectif retenu n'est pas de revenir sur les fonctions et compétences du tuteur, mais de proposer des repères pour la compréhension du processus d'interrelation par lequel l'apprenant avec des tiers que sont les autres acteurs (tuteurs, pairs) et les choses (l'artefact), sollicite et élabore des formes tutorales.

Nous proposons plus spécifiquement de traiter de l'hypothèse selon laquelle l'ouverture d'un dispositif de formation, et les pratiques tutorales associées, supposent l'initialisation d'un processus de coconstruction intentionnelle et régulé qui sollicite la création d'un espace intersubjectif de coinvestissement (Pineau et Legrand, 1993) organisateur d'un espace d'interaction transactionnel et transitionnel.

Après avoir rappelé l'évolution des relations qui unissent les acteurs d'un dispositif de formation ouverte et à distance, nous proposerons une matrice d'analyse du processus d'actualisation du dispositif. Les exemples proposés issus d'observations conduites auprès d'étudiants et d'adultes en reprise d'études concrétisent cette construction et étayent l'hypothèse de l'existence d'une zone proximale qui organisent l'actualisation du dispositif prescrit en un dispositif vécu. Nous poursuivrons notre propos en explicitant la structuration temporelle de cette dynamique selon trois phases. Nous conclurons cet article par l'une des caractéristiques de cet espace d'interaction : la fonction contenante et transitionnelle de l'espace d'interaction.

\section{L'implication de l'apprenant : forme d'exercice du pouvoir et de reconnaissance}

La rupture de l'unicité de «l'espace-temps social » conventionnel générée par la mise à distance des acteurs de l'acte de formation suppose la reconfiguration d'une structure organisatrice et contenante de l'acte éducatif et de l'apprentissage. Classiquement, l'ouverture est présentée dans la culture francophone comme une rupture des unités de temps, de lieu et d'action. L'idée principale à retenir est l'évolution des règles et schèmes d'actions conventionnels qui structurent habituellement la formation. Evolution, voire innovation qui sollicite chez l'apprenant et le formateur des compétences spécifiques. Interprétée sous l'angle des interactions entre acteurs, la triangulation initiale (Houssaye, 1988) qui réunit apprenant/savoir/formateur évolue vers un système plus complexe. Cette complexité naît de la multiplicité des acteurs et de leurs interrelations: sujet/outil/ objet/communauté/règles/division du travail (Kuutti, 1991 ; Engeström, 1987). Cette évolution, inscrite en continuité des travaux relatifs aux théories de l'activité, au socio-constructivisme, (Vygostky, 1978; Leontiev, 1984) est principalement marquée par la centration sur l'apprentissage considéré comme transformation de l'environnement consécutive à la réalisation de la tâche. La tâche n'est pas la finalité, mais bien une modalité de réalisation d'un nouvel état de soi, de l'autre, et des choses. Dans un tel contexte, il devient difficile de considérer le tutorat dans ces 
dispositifs uniquement comme un processus de rationalisation de l'acquisition de savoirs par le sujet apprenant. La prégnance de la dimension intentionnelle de l'acte d'apprendre supplante la simple "fonctionnalisation» du tutorat. L'initialisation d'une « dynamique intentionnelle » (Linard, 2001) qui va servir de moteur à l'action devient dès lors l'une des préoccupations premières des concepteurs et prescripteurs de tels dispositifs. L'intention d'agir, préalable à la motivation d'accomplissement, apparaît comme vecteur essentiel de l'apprentissage.

Les dispositifs de formation ouverte et à distance (DFOAD) offrent un ensemble de fonctionnalités, de ressources matérielles et humaines dont l'objectif est de contribuer à la réalisation de l'apprentissage. Ils constituent un potentiel de situation (Jullien, 1996) dont l'actualisation par les acteurs contribuera à la réalisation du projet d'apprentissage. De même que tous les individus d'un groupe classe ne participent pas de la même manière à un cours en présentiel, l'implication des apprenants à distance se traduit par des organisations singulières du dispositif initial. Cette implication est d'ailleurs sollicitée par les concepteurs de ces dispositifs, qui dans une mouvance économique des biens et des services attendent de l'apprenant une posture active et participante. Ce dernier est fortement invité par les concepteurs, prescripteurs et formateurs à participer à la coconstruction des savoirs, dynamique pour laquelle ces derniers organisent et mettent à disposition un ensemble de ressources (concept de servuction ${ }^{1}$ ).

Au-delà de cet attendu, il convient de comprendre le processus réel d'implication de l'apprenant, préalable à la définition des postures tutorales. Pour ce faire nous proposons d'analyser cette dynamique en considérant que l'apprentissage dans des dispositifs de formation ouverte et à distance sollicite l'apprenant dans un double processus : la coconstruction du dispositif et des savoirs (Paquelin et Choplin, 2003). Cette étude conduite auprès d'élèves ingénieurs en formation initiale a explicitement mis en évidence les multiples formes de régulations sollicitées pour actualiser le dispositif prescrit : régulation auto-référentielle, régulation par et avec les pairs, régulation par et avec le tuteur.

Pour comprendre cette dynamique, nous nous appuierons sur des éléments issus de la psychologie du travail. Les travaux antérieurs conduits par (Samurçay et Pastré, 1995; Rabardel, 1995), ont avancé la conclusion d'une dynamique d'appropriation (genèse instrumentale pour Rabardel) qui différencie un état prescrit d'un état réel. Le processus de genèse instrumentale réalisé par l'individu lui permet de construire activement ses schèmes d'utilisation, soit en réinvestissant des schèmes familiers, déjà constitués, soit en produisant de nouveaux schèmes qui lui permettent d'atteindre les buts visés (Docq et Daele, 2003). Nous proposons d'étudier l'appropriation des dispositifs de formation ouverte et à distance dans cette continuité pour comprendre les postures tutorales sollicitées et proposées. Ainsi,

1. Albertini J.-M., Une nouvelle économie de la formation? in Etudes et expérimentations en formation continue, $\mathrm{n}^{\circ}$ 16, La Documentation Française, juillet-octobre 1992, Paris, p. 3-8. 
nous faisons l'hypothèse que cette dynamique de coconstruction relie quatre « états » du dispositif : l'état prescrit, l'état perçu, l'état prévu et l'état vécu.

Nous nommons l'état prescrit (EP), le dispositif tel qu'il est conçu par les concepteurs et porteur d'une prescription ou intention d'usage. Le sujet construit des représentations du monde. Ces constructions intellectuelles momentanées permettent de donner sens à une situation en utilisant les connaissances stockées en mémoire et/ou les données de l'environnement, dans le but «d'attribuer une signification d'ensemble aux éléments issus de l'analyse perceptive " (Richard, 1990). L'état perçu (EPe) est un construit par l'apprenant, qui à partir d'indices sémiotiques, de sa représentation de ce type de dispositif et de la tâche à accomplir, et de ses besoins élabore une image du dispositif (image opérative, Ochanine, 1978). Les représentations agissent comme des schèmes qui dirigent l'action du sujet. Elles sont circonstanciées et finalisées par la tâche ou la décision à prendre. Lors d'une étude consacrée à l'appropriation d'un dispositif d'apprentissage coopératif à distance par des formateurs en soins infirmiers, nous avons observé que la pratique de modification de l'objet d'autrui (exemple: une production textuelle) n'est pas inscrite dans les pratiques professionnelles, qui au contraire sont davantage structurées vers une construction d'un dossier commun (exemple dossier du patient) par compilation d'informations (prescription, réalisation d'actes, etc.). De même la traçabilité informationnelle qui traduit le suivi des différents actes réalisés, critère de qualité de l'acte professionnel, pourrait venir en contradiction avec la perception que les apprenants ont de la perte des informations échangées et produites dans de tels dispositifs. Cet exemple illustre l'effective influence des représentations et de l'identité de l'apprenant sur la perception et l'acceptation de l'état prescrit.

L'état prévu (EPr) est une situation projective dans laquelle l'apprenant envisage de réaliser son apprentissage. Il exprime l'organisation spatio-temporelle, les activités et les relations qui organisent le projet de dispositif.

L'état vécu (EVe) ou réel est l'actualisation effective du dispositif prescrit, ce qui est réellement utilisé. Cette distinction propose un premier cadre d'explication du processus d'actualisation du dispositif initial et montre ainsi l'écart entre l'offre et l'usage. Si l'apprenant construit une représentation du dispositif, le tuteur investit le dispositif prescrit de sa propre intention qui semble être pour partie déterminée par l'image qu'il a de la fonction tutorale. Ainsi, apparaît potentiellement un ensemble de tensions entre l'état perçu des apprenants et celui des tuteurs. Ce premier niveau est complété par d'éventuelles tensions établies entre les différents états prévus, ceux des apprenants et ceux des tuteurs. Pour comprendre cette dynamique, il importe d'identifier l'ensemble des catégories d'acteurs engagés dans un tel projet afin de gérer au mieux la distance transactionnelle (Moore, 1996), c'està-dire les effets du dialogue et de la structure d'aide sur l'apprentissage à distance. 
Cette distinction entre le prescrit, le perçu, le prévu et le réel ${ }^{2}$ est l'expression même de l'ouverture d'un dispositif. Certains auteurs parlent de plasticité des dispositifs. Ce sont des dispositifs pour lesquels les concepteurs ont cherché à anticiper l'action en situation (Béguin, Cerf, 2004). Le qualificatif «ouvert» suppose d'une part qu'il y ait une flexibilité potentielle de certaines composantes du dispositif, et que d'autre part des choix puissent être effectués par l'apprenant. Ces premiers éléments descriptifs supposent l'actualisation du couple pouvoir/reconnaissance :

- la reconnaissance de ce qui est actualisable, c'est-à-dire le passage d'un état virtuel à un état effectif, réalité tangible du dispositif ;

- le pouvoir d'agir et plus spécifiquement de définir la valeur des variables de certains paramètres du dispositif initial.

Cette prise de pouvoir sur le dispositif prescrit, considérée comme exercice de l'autonomie, donne naissance au dispositif réel et peut s'analyser en différenciant :

1) ce qui est actualisé en l'état ;

2) ce qui n'est pas actualisé ;

3) ce qui est transformé ;

4) ce qui est créé.

Cette proposition rejoint les travaux de Henderson et Kyng (1991) relatif aux niveaux de transformation des systèmes. Ces auteurs distinguent ce qui est non modifiable, modifiable et adaptable, transformable. Cette distinction suppose la définition de degré de liberté d'action au sein même du dispositif prescrit mais également l'identification de zone d'ancrage ${ }^{3}$ de ce dispositif à des éléments externes. Giordan et De Vecchi (1987) ${ }^{4}$ proposent la métaphore de l'ancrage comme illustration de la dynamique d'apprentissage. Les conceptions préalables de l'apprenant, ce qu'ils nomment le « déjà-là », constituent les «structures d'accueil » qui permettent de fédérer de nouvelles informations. Ces éléments peuvent être identifiés et catégorisés par leur existence préalable. L'antériorité des pratiques de formation, l'appartenance à un réseau social, la maîtrise d'outils technologiques, cognitifs, structurent ce préalable ou «déjà-là » qui oriente la dynamique intentionnelle d'appropriation du dispositif prescrit. Reconnaître à l'apprenant le pouvoir d'actualisation de tout ou partie du dispositif requiert l'acceptation par le tuteur, l'institution, d'apports d'éléments externes (exemple : l'apprenant mobilise des outils de communication qui n'appartiennent pas au dispositif prescrit par les concepteurs et/ou tuteurs). Ce potentiel actualisable par l'apprenant, seul ou

2. En référence à la différenciation établie par les psychologues du travail entre le travail prescrit et au travail réel (Pastré, 1999).

3. Cette notion de « zone d'ancrage » fait référence aux travaux de Giordan relatifs au modèle allostérique de l'apprentissage.

4. Giordan A., De Vecchi, G., Les origines du savoir, Delachaux \& Niestlé, 1987. 
accompagné, définit l'ouverture du dispositif comme la zone d'exercice de son autonomie.

Accepter cette dynamique revient à reconnaître et accepter le pouvoir d'inventivité de l'apprenant (Weill-Fassina et al., 1993). Ces auteurs rappellent que «les actions ne sauraient être réduites à l'effection de réponses à des stimuli reçus, plus ou moins passivement [...] les opérateurs explorent, interprètent, utilisent, transforment leur environnement technique, social et culturel ». Cette inventivité 5 exprimerait ce que nous proposons de nommer la genèse dispositive (Paquelin, 2003). Ce processus est défini comme l'actualisation "écologique» des composantes du dispositif prescrit qui par recombinaison d'éléments issus d'espaces-temps sociaux hétérogènes, initie et régule un dispositif vécu. L'écologie est retenue dans sa dimension composite en cela qu'elle résulte de la convergence de zones d'acceptation des différentes catégories d'acteurs engagés dans ce processus : l'institution, les tuteurs, les apprenants. Cette genèse est une coconstruction régulée et régulatrice.

Le développement de cette autonomie sollicite alors une double boucle réflexive, celle de l'apprentissage et celle de la métacognition. Le formateur, devenu tuteur, facilitateur, animateur, accompagnateur, agit, par des postures et compétences appropriées, en vue de l'émergence de sens, tant au niveau des savoirs que du savoir apprendre. La gestion de l'ouverture devient possible, pour le tuteur, dès lors qu'il admet que le sens, celui du projet, précède la connaissance. Une telle posture suppose d'accepter qu'il y ait des savoirs qu'il ne maîtrise pas, ceux que l'autre construit et la manière dont il les construit. Pour initier et développer ce processus d'appropriation, nous observons une triple posture tutorale : proactive, coactive et réactive.

En résumé, la genèse dispositive par laquelle l'apprenant et le collectif d'acteurs actualisent les fonctionnalités et ressources initiales introduit dans le dispositif prescrit initial des éléments nouveaux (outils, ressources informationnelles, etc.). Cette genèse traduit une dynamique d'appropriation qui engage l'ensemble des acteurs dans leur dimension existentielle. Pour Honoré (2001), «S'approprier signifie intégrer quelque chose dans son expérience (un fait, un événement, une situation, une connaissance, une technique...) par sa compréhension, donc par le sens qui lui est donné, en le rapportant à ce qui nous concerne, à ce qui nous soucie. L'appropriation de ce que nous comprenons est rendue possible par son explication qui la rapporte à des significations préalablement acquises en les confirmant, en les transformant ou en les complétant par de nouvelles significations. " La fonction tutorale assure les nécessaires ajustements entre le dispositif prescrit et le contexte de réalisation du projet de formation. Cette finalité présentée, il convient d'en comprendre la dynamique et le processus.

5. Cette inventivité est à relier avec la métis grecque, forme d'intelligence rusée engagée dans la pratique. 


\section{Les degrés de liberté d'action : reconnaître son pouvoir d'agir}

\section{Proposition d'un cadre d'analyse de l'appropriation}

La flexibilité, plasticité d'un dispositif de formation ouverte se concrétise par l'existence de degrés de liberté d'action offerts à l'apprenant. Dans un système, ces degrés de liberté d'action sont définis pour chaque caractéristique. Dès lors que ces dernières sont précisées pour un dispositif de formation ouverte, les degrés de liberté d'action sont alors explicités. Quelle modélisation choisir? A ce stade de notre construction $^{6}$, nous retiendrons les composantes suivantes pour définir un tel dispositif $^{7}$ :

1) les objectifs, les savoirs, les compétences visées ;

2) les activités d'apprentissage ;

3) les activités d'évaluation ;

4) les ressources informationnelles didactisées ou non ;

5) les activités de régulation;

6) les activités de production ;

7) les activités de planification, de gestion et de suivi du parcours et des activités.

La dynamique d'appropriation exposée au début de cet article et cette proposition de catégorisation permettent de construire une matrice d'analyse de l'exercice du couple «pouvoir/reconnaissance » (cf. tableau 1). Chaque intersection est l'objet d'une analyse spécifique qui explicite la dynamique en prenant en compte ce qui revient à l'apprenant, au dispositif, à l'environnement ou au contexte. Pour affiner l'analyse, nous proposons de définir le degré d'actualisation des fonctionnalités comme suit :

- fonctionnalité jamais actualisée (degré 0),

- fonctionnalité actualisée puis abandonnée (degré 1),

- fonctionnalité partiellement actualisée (degré 2),

- fonctionnalité intégralement actualisée (degré 3),

- fonctionnalité transformée (degré 4),

- fonctionnalité créée (degré 5).

Le tuteur devient celui qui accompagne l'apprenant dans cette dynamique d'appropriation avant même d'être celui qui accompagne les apprentissages.

6. Cette catégorisation n'a pas de caractère exhaustif ni définitif, sa fonction est de soutenir l'argumentation. Elle se rapproche des propositions de Paquette (2002).

7. Pour plus d'informations, le lecteur se réfèrera aux publications relatives à la grille GENIP (grille d'évaluation du niveau d'individualisation des programmes) et à la thèse d'A. Jezegou qui réactualise les paramètres de cette grille (Université Paris X Nanterre, soutenue le 9 juin 2004). 


\begin{tabular}{|l|l|l|l|l|l|}
\cline { 2 - 5 } \multicolumn{1}{c|}{} & \multicolumn{2}{c|}{ Non-actualisation } & Actualisation & Transformation & Création \\
\cline { 2 - 5 } \multicolumn{1}{c|}{ Inconsciente } & Consciente & & & \\
\hline $\begin{array}{l}\text { Activités } \\
\text { d'apprentissage }\end{array}$ & & & & & \\
\hline $\begin{array}{l}\text { Activités } \\
\text { d'évaluation }\end{array}$ & & & & & \\
\hline $\begin{array}{l}\text { Ressources } \\
\text { informationnelles }\end{array}$ & & & & & \\
\hline $\begin{array}{l}\text { Activités } \\
\text { et outils de } \\
\text { communication }\end{array}$ & & & & & \\
\hline $\begin{array}{l}\text { Activités et outils } \\
\text { de production }\end{array}$ & & & & & \\
\hline $\begin{array}{l}\text { Activités et outils } \\
\text { de planification }\end{array}$ & & & & & \\
\hline
\end{tabular}

Tableau 1. Matrice d'analyse de la dynamique d'appropriation

\section{Exemple de dynamique d'appropriation}

L'exemple qui suit est issu de l'analyse d'un dispositif d'apprentissage coopératif qui mobilise une plate-forme de travail coopératif (COOPERA) ${ }^{8}$. Le public cible est constitué d'adultes en formation continue. La structure de la tâche proposée aux apprenants mobilisait les principales fonctionnalités de la plate-forme (cf. tableau 2). Les conclusions qui suivent concernent plus particulièrement l'actualisation des fonctionnalités de la plate-forme par les apprenants dans la perspective de réalisation d'une tâche en commun.

\begin{tabular}{|c|c|}
\hline Structure de la tâche & Fonctionnalités de COOPERA \\
\hline Organisation & Calendrier (0) \\
Recherche documentaire & Stockage (3) \\
Analyse & Mettre à jour (2) \\
Débat & Communiquer (1) \\
Production & Publier (3) \\
\hline
\end{tabular}

Tableau 2. Structure de la tâche - fonctionnalités de COOPERA (le numéro qui suit l'intitulé de la fonctionnalité exprime de degré d'actualisation)

8. Projet soutenu dans le cadre de l'action RIAM-CNC. 
Les observations montrent que si la tâche a été réalisée avec satisfaction du résultat obtenu, le potentiel de l'artefact a été partiellement actualisé. Quatre situations ont été relevées définissant ainsi quatre degrés d'actualisation :

- fonctionnalité jamais actualisée (degré 0),

- fonctionnalité actualisée puis abandonnée (degré 1),

- fonctionnalité partiellement actualisée (degré 2),

- fonctionnalité intégralement actualisée (degré 3).

Ces degrés d'actualisation peuvent être interprétés selon les trois principes ${ }^{9}$ qui entrent en jeu dans la production par le sujet de ses schèmes d'utilisation d'un outil (Rabardel, 1995) : le principe d'économie, la recherche d'efficacité, l'atteinte d'un équilibre dans l'outillage. Cette dynamique résulte de perceptions et d'actions de la communauté d'acteurs engagés dans la réalisation de la tâche : tuteur, apprenants.

Ainsi l'actualisation des fonctionnalités de la plate-forme de travail coopératif s'opère selon une dynamique en quatre temps :

- temps 1 : temps de la découverte des fonctionnalités,

- temps 2 : sélection des fonctionnalités reconnues comme pertinentes,

- temps 3 : temps de test, de rodage des fonctionnalités,

- temps 4: temps de confirmation/infirmation de l'actualisation des fonctionnalités.

Dans le cas où l'actualisation de la fonctionnalité n'est pas maintenue, le sujet procède ou non à une substitution par un autre outil. Dans le cas étudié, les outils de communication ont été abandonnés au profit d'autres outils : la messagerie, le chat. La messagerie interne à la plate-forme ayant été perçue comme superflue et moins facile à utiliser que les messageries traditionnelles (principe d'économie). Les participants à l'expérimentation ont jugé qu'il était plus simple d'utiliser leur propre outil de messagerie électronique et ne souhaitaient pas multiplier leur outil d'échange asynchrone.

Le non-usage des outils d'organisation que sont l'agenda et le gestionnaire de tâches relève à la fois de ce principe d'économie, mais également du principe d'efficacité. Le principe d'organisation préalable de la coopération est reconnu dans sa pertinence et sa nécessité. Chaque groupe a mis en place des outils spécifiques de

9. Le principe d'économie : l'individu a tendance à choisir l'outil qu'il connait le mieux, ou l'outil le plus disponible, et s'en servir pour le maximum de tâches, pour économiser l'énergie qui devrait être fournie pour s'approprier et/ou acquérir un autre outil. La recherche d'efficacité : si l'outil proposé ne lui paraît pas le plus efficace au vu des objectifs à atteindre, l'individu va soit choisir un autre outil, soit utiliser l'outil proposer d'une façon inattendue des concepteurs (usages informels). L'atteinte d'un équilibre dans l'outillage: le sujet restructure l'outillage dont il dispose en fonction de son expérience. Il organise les outils, leurs usages formels et informels, de manière à atteindre un bon équilibre entre principe d'économie et recherche d'efficacité. (Rabardel, 1995). 
gestion du travail à distance et a demandé à ce que le premier scénario pédagogique soit modifié pour intégrer un temps d'organisation présentiel préalable à l'intersession.

Malgré les difficultés de prise en main ${ }^{10}$, le questionnement proposé a posteriori montre que la représentation de la plate-forme est proche des fonctionnalités définies par les concepteurs :

- un outil de mise en commun de productions,

- un moyen de constitution d'une base de données d'informations,

- un moyen d'échanges de documents (mutualisation),

- un moyen de suivi des travaux, de repérage dans l'avancement des productions.

Sans revenir sur les développements précédents, l'actualisation des fonctionnalités semble déterminée pour partie par le contexte d'usage et traduit le degré d'efficience acceptable par les uns et les autres. Dans le cas observé l'efficience semble déterminée par l'accessibilité des équipements, les compétences à développer et le temps disponible au niveau du travail individuel et collectif. La fonction tutorale est sollicitée par les apprenants pour définir l'acceptabilité des modifications souhaitées, maintenir le degré d'exigence de la production attendue et pour réguler les relations entre les membres du groupe.

\section{L'appropriation : dynamique d'efficience contextualisée}

Dans d'autres situations observées, les ressources informationnelles proposées ne sont pas utilisées malgré l'actualisation des activités d'apprentissage. Les apprenants n'actualisent pas les ressources et mobilisent des ressources extérieures au dispositif. Ce choix n'est pas fait au hasard. Il est consécutif à une dynamique de reconnaissance qui les a conduits à identifier comme utilisables et potentiellement efficaces des ressources informationnelles maîtrisées en dehors du dispositif prescrit (Paquelin, 2002). Leur pouvoir de choisir doublé de leur capacité à reconnaître une ressource adaptée leur permet «d'inventer», de recréer un dispositif réel qui correspond d'une part à leur pratique d'apprentissage, à ce qui est attendu comme performances dans le dispositif prescrit et ce qui convient à leur environnement d'apprentissage.

Notons que la non-actualisation d'une composante du dispositif prescrit peut résulter d'une omission, elle est alors qualifiée de non-actualisation inconsciente. L'intervention réactive d'un tuteur, qui reconnaît l'origine de la non-actualisation permettra à l'apprenant d'une part de prendre conscience de cette omission et

10. Il convient de rappeler que ces résultats font suite à l'utilisation de la première version de COOPERA, version non finalisée et objet de la recherche. 
d'autre part de réagir en conséquence. Son intervention est également de nature proactive en cela qu'il permet à l'apprenant de découvrir l'ensemble des ressources proposées par le dispositif prescrit. Dans de nombreux cas de figure l'actualisation conjointe de certaines fonctionnalités traduit une posture coactive (exemple : tuteurs et apprenants décident ensemble d'utiliser un chat pour échanger dans une perspective de régulation).

Ce cadre d'analyse permet de comprendre en quoi cette dynamique est située (Suchman, 1987), c'est-à-dire qu'elle est fonction du contexte d'usage et donc non réductible à des schémas préétablis, délimitant ainsi «la fraction» industrialisable du dispositif et du tutorat. La variabilité de l'actualisation d'un dispositif ne saurait être infinie. Il conviendrait de préciser la part « irréductible » de la variabilité, celle qui définit une constance d'actualisation nécessaire au maintien d'un degré minimal d'efficacité du dispositif. La dimension sociale nécessaire à tout apprentissage dans un contexte institué suppose qu'un seuil minimal commun d'actualisation des fonctionnalités du dispositif soit atteint par l'ensemble des acteurs engagés dans le processus de formation. Au cours d'une étude antérieure ${ }^{11}$, nous avons observé que ce seuil minimal était l'actualisation de la fonction de socialisation des productions des différents groupes d'apprenants. Le dispositif prescrit serait une œuvre inachevée sollicitant l'apprenant pour une finalisation singulière et temporaire dans une zone d'acceptabilité. Daniellou (2004) parle de flexibilité synchronique pour exprimer des systèmes qui laissent des «marges de manœuvre» aux utilisateurs. Pour cet auteur "l'enjeu de l'approche de l'activité future n'est pas de prévoir en détail l'activité qui se déroulera dans l'avenir, mais de prévoir l'espace des formes possibles d'activité future». Cette plasticité assure une double fonction d'adaptation: adaptation situationnelle ou située, adaptation personnelle. Le dispositif est adaptable à la situation dans laquelle l'apprenant réalise en fonction de ses propres capacités son apprentissage. Elle permet une actualisation qui prend en compte le potentiel de situation dans laquelle se situent l'apprentissage et les capacités de l'apprenant à réaliser son apprentissage.

Cette adaptation ne peut se réduire à la simple substitution d'un élément par un autre. Elle peut être l'objet d'apprentissages parfois complexes pour l'apprenant et le tuteur. Cela nécessite d'être en capacité de créer des liens entre des éléments issus d'espaces hétérogènes.

Comment s'organise l'acceptabilité d'éléments extérieurs au dispositif prescrit? Existe-t-il des limites à ces reconfigurations? Quels sont les vecteurs qui maintiennent l'état effectif et productif du dispositif nouvellement configuré ? Quelles sont les formes et postures tutorales sollicitées et par qui sont-elles assurées? Autant de questions qui demandent de multiples approfondissements.

11. Etude COOPERA. 


\section{L'appropriation : un processus intermonde}

La zone d'acceptabilité peut s'analyser comme étant la zone de gestion de tensions entre différentes composantes (cf. figure 1). Cette appropriation se traduit par la coconstruction d'un dispositif singulier qui résulte pour chaque apprenant d'un ensemble de négociations. L'émergence même de ce dispositif singulier est l'expression du lieu d'exercice de pratiques d'autoformation. En son absence, ces pratiques ne pourraient pas se développer, confinant le sujet apprenant dans une situation de formation plus conventionnelle. Pour tenter de cerner ces processus de régulation tout en réduisant la complexité du système, nous traiterons dans ce qui suit, de ce qui concerne plus directement l'apprenant.

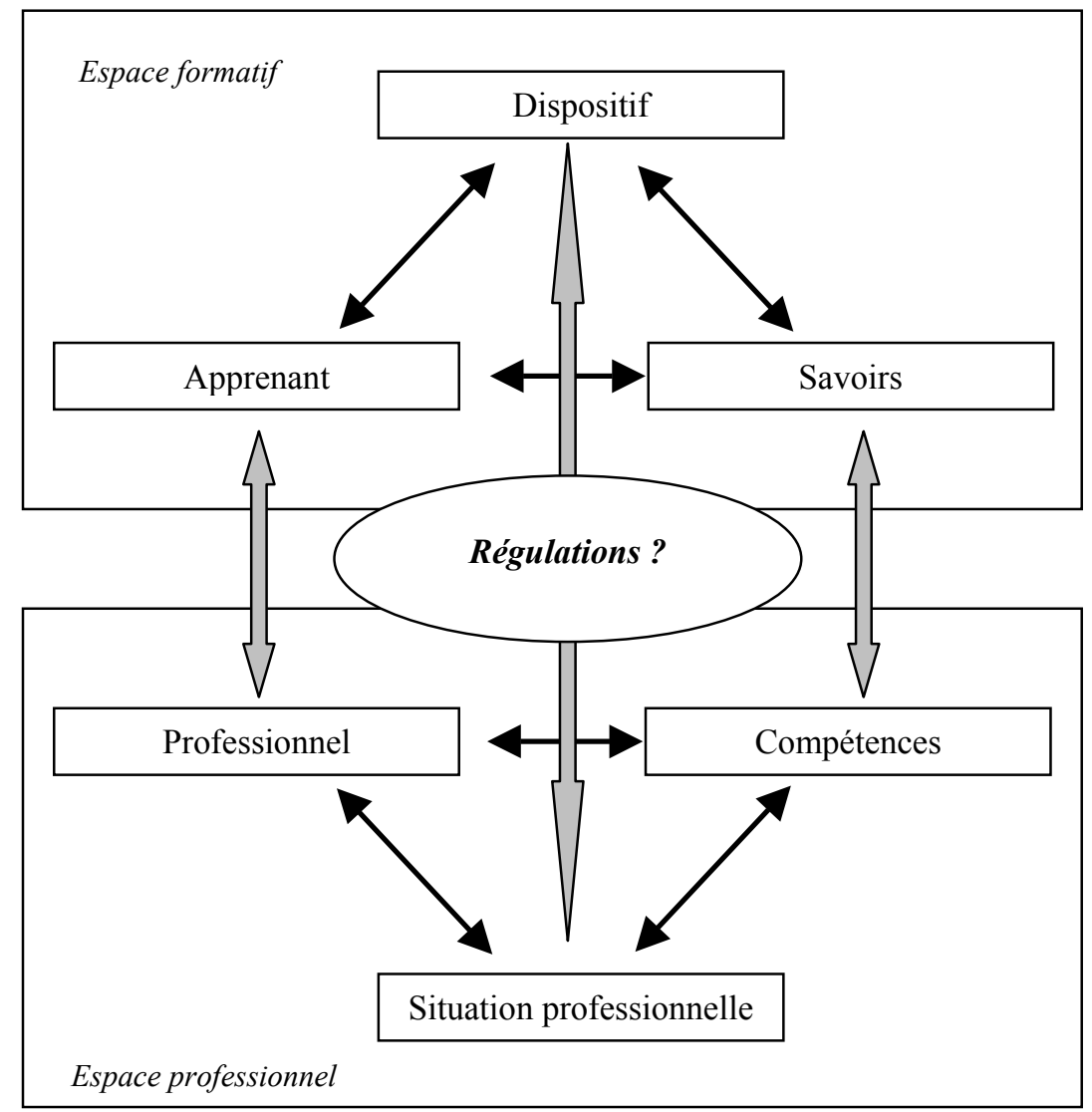

Figure 1. L'appropriation, l'enjeu des régulations

Les observations conduites auprès d'élèves ingénieurs et de professionnels en situation de travail montrent qu'une dynamique relie deux espaces-temps sociaux : 
celui de l'apprentissage, celui de la sphère privée et/ou professionnelle. Cette appropriation peut être décrite selon deux dimensions : le niveau interne à chaque espace, le niveau externe qui relie deux espaces. Considérons que la mise en place d'une action de formation s'inscrit dans un espace spécifique que nous nommerons l'espace formatif. Cet espace formatif, est dans le cas d'une formation professionnelle distinct de l'espace professionnel qui est le lieu d'exercice des compétences du sujet. Après avoir explicité chacun de ces espaces, nous traiterons de leur articulation.

L'espace-temps d'apprentissage ou espace formatif est déterminé par trois composantes que sont l'apprenant, le savoir à acquérir et le dispositif prescrit. Les observations réalisées montrent combien les régulations sont nombreuses et orientées vers la recherche d'un équilibre, d'un état stable que définit un rapport coût/efficacité acceptable par l'apprenant, pour lui-même et dans la perception qu'il a, à la fois des attentes de son contexte institutionnel et des degrés de liberté qui lui sont offerts.

L'espace professionnel met en relation le sujet en tant que professionnel, les compétences maitrisées et à construire, et la situation professionnelle actuelle et en devenir. C'est par la relation aux deux autres composantes que le sujet définit son identité professionnelle et ses attentes en matière de formation. La dimension temporelle est éminemment présente car elle définit en partie la performance attendue par l'organisation.

Pris séparément, chaque espace est le lieu de vie de deux systèmes spécifiques mus par leur propre finalité et qui possèdent leur propre processus de régulation. La mise en place d'un dispositif de formation ouverte et à distance introduit une interaction forte entre ces deux espaces. L'analyse de ces interactions semble expliquer, du moins en partie les déterminants de l'appropriation d'un dispositif de FOAD par des sujets en situation d'apprentissage.

Il semblerait que cette appropriation soit déterminée par les tensions entre ces deux espaces. Dans le cas d'apprenants en situation de formation initiale, ces deux espaces sont confondus, du moins les écarts sont réduits. L'espace professionnel serait l'espace privé, celui au sein duquel se déroulent les pratiques cachées de formation. L'étudiant trouverait dans une situation de FOAD, un espace d'expression de la globalité de son identité d'apprenant tendant ainsi à une convergence de deux postures, d'une part celle de l'étudiant «public» et d'autre part, celle de l'étudiant "caché». De même, les pôles savoir et compétences seraient confondus. Quant au dispositif de l'espace formatif il serait en tension avec la situation professionnelle, qui dans le cas de la formation initiale serait le dispositif caché, les pratiques non formelles habituelles des étudiants. En conséquence, l'ouverture de la formation, tendrait à réduire les tensions entre ces deux pôles jusqu'à confondre ces deux espaces.

La situation observée dans le monde professionnel est bien différente. La difficulté à s'approprier l'espace formatif tient probablement aux tensions 
binomiales importantes. La recherche de construction de compétences semble déterminer le rapport au savoir qui ne serait accepté que dans la perspective de sa mobilisation en situation professionnelle. Ceci se traduit par la recherche de situation de formation finalisée, et par la quête de mise en relation de savoirs épars acquis en situation professionnelle. Le dispositif proposé, et notamment l'absence de cadre d'action explicite rendrait difficile l'articulation entre celui-ci et les contraintes de la situation professionnelle qui demande une productivité. Enfin, la représentation que les professionnels construisent de l'apprenant que le dispositif leur demande d'être est rejetée car jugée trop scolaire. Tous ces éléments agissent pour mettre en opposition ces deux espaces.

\section{L'hypothèse d'une zone proximale d'actualisation}

Le cadre présenté dans cet article considère l'appropriation comme principe directeur de l'apprentissage dans des dispositifs de formation ouverte et à distance. Il est bien entendu que cette appropriation s'effectue dans un contexte défini notamment, par des règles qui régissent et contiennent l'acceptabilité des degrés de liberté d'action proposés aux acteurs du dispositif.

Nous posons l'hypothèse que cette dynamique actualisante du dispositif prescrit est contenue dans une zone proximale d'actualisation $(\mathrm{ZPA})^{12}$, zone délimitée par l'acceptabilité négociée ou non (tout n'étant pas négociable) entre les différentes catégories d'acteurs de l'actualisation.

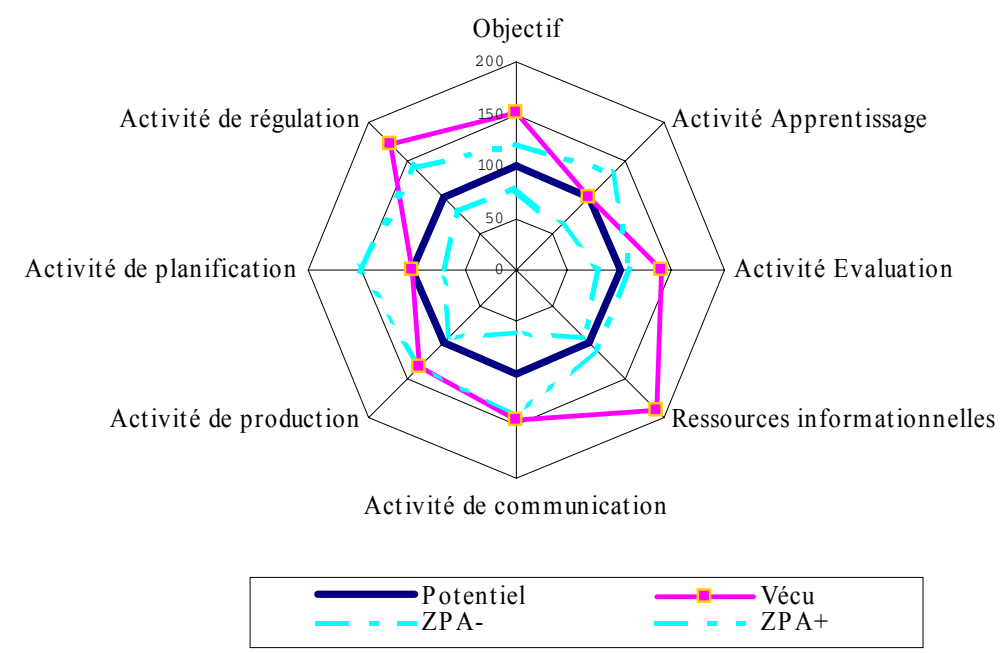

Figure 2. La zone proximale d'actualisation

12. Cette proposition s'inspire de la zone proximale de développement. 
L'hypothèse est ainsi formulée : l'appropriation d'un dispositif traduite par sa reconfiguration mise en contexte, est réalisée dans une zone proximale d'actualisation à laquelle participent de multiples acteurs que sont l'apprenant, les autres et les choses. Cette zone est définie à partir d'un ensemble de paramètres constitutifs du dispositif (cf. supra). La dimension proximale est fournie par les degrés d'ouverture exprimés en degrés de liberté d'actualisation, qu'ils soient explicites ou implicites. Cette proposition conduit à penser l'actualisation comme un déplacement au sein d'un espace défini par deux zones situées de part et d'autre d'une forme optimale et référentielle du dispositif prescrit. Cet espace détermine l'ouverture prescrite, autorisée par les concepteurs et prescripteurs. Ces limites supérieures $(\mathrm{ZPA}+)$ et inférieures (ZPA-) concrétiseraient la zone d'acceptation, d'exercice de la liberté de choix (cf. figure 2). L'actualisation traduirait ce territoire « habité » par les acteurs engagés dans l'actualisation du dispositif et au sein duquel se déroulerait le tutorat.

L'acceptabilité exprimerait la gestion des écarts et tensions entre le prescrit et le perçu, voire le désiré. Cette zone proximale d'actualisation assure une fonction transactionnelle et transitionnelle.

La dynamique d'appropriation d'un dispositif de formation ouverte ne semble pas de type linéaire, mais de type spiralé. La première étape est celle de la reconnaissance de l'offre au cours de laquelle l'apprenant reconnaît certaines composantes du dispositif. L'apprenant teste l'efficience du dispositif pour ensuite retenir ce qui lui semble pertinent pour la suite de son projet.

Ces différents ajustements qui conduisent à des états stabilisés permanents ou temporaires d'un dispositif vécu sont l'objet de régulations entre l'apprenant et les autres membres engagés dans le dispositif: les tuteurs et les autres apprenants (Paquelin et Choplin, 2003). Ces régulations ont pour objet de maintenir l'évolution du dispositif prescrit dans son aire de développement.

\section{L'accompagnement de l'appropriation : mise en lien, mise en sens}

Ainsi présenté, l'apprentissage dans un dispositif de formation ouverte et à distance sollicite des fonctions tutorales qui accompagnent l'actualisation des composantes du dispositif par l'apprenant, le groupe d'apprenants.

Il est bien entendu que l'apprenant, de par ses caractéristiques personnelles cognitives, émotionnelles, affectives, sera plus ou moins en capacité d'agir de manière autonome. Les travaux de recherches relatifs à l'étude des apprenants dans ces dispositifs montrent combien certaines caractéristiques de leur situation personnelle, professionnelle interviennent dans ces dynamiques d'appropriation (citons par exemple le sentiment d'urgence et/ou de nécessité, Glikman 2002). Lors de l'étude d'une situation d'apprentissage coopératif, nous avons observé que la dématérialisation de l'information et des supports génère la construction d'une 
représentation tridimensionnelle de circulation de l'information. Les résultats observés auprès d'adultes en reprise d'études corroborent ceux obtenus avec les enfants dans l'expérimentation conduite avec le même dispositif technologique. L'ergonomie de surface et de profondeur de l'artefact ne permet pas dans la version utilisée d'exercer un étayage dans la compréhension de la circulation de l'information et des échanges. L'analyse ergonomique conforte les propos des apprenants qui ne peuvent construire rapidement une représentation mentale des échanges, et par conséquent n'actualise pas certaines fonctionnalités. Cette construction mobilise notamment les capacités de l'apprenant à se représenter l'espace non pas comme un ensemble géométrique euclidien qui affecte à tout point des coordonnées, mais comme un espace topologique au sein duquel chaque élément est défini par rapport aux relations qui l'unissent à d'autres éléments (Fastrez, 2002). La capacité de l'apprenant à établir des liens entre les différentes dimensions du dispositif semble participer à l'actualisation de fonctionnalités de ce dernier.

La reconnaissance par l'utilisateur des éléments et des liens qui les unissent suppose une forte utilisabilité, et une haute affordance ${ }^{13}$ de l'application (ergonomie de surface, ergonomie de profondeur). Identifier cet espace, revient à reconnaître les frontières à l'action (Vicente, 1999). Cette perception est d'autant plus importante qu'elle agit comme forme organisatrice de l'activité de l'apprenant, qui selon son pouvoir d'action suscitera plus ou moins de guidage. Ainsi l'activité d'apprentissage est sous l'influence d'une double détermination: celle du dispositif et celle de l'apprenant. Elle est sous-tendue par une construction sociale du sujet apprenant qui participe à l'actualisation du dispositif prescrit.

\section{Le cycle d'actualisation du dispositif et de construction des apprentissages}

Trois phases peuvent être identifiées pour comprendre et interpréter cette dynamique : une phase d'implication et d'engagement, une phase de distanciation, une phase de transformation. Ces trois temps sont l'objet d'une analyse particulière pour tendre vers une explicitation argumentée de cette dynamique et comprendre comment se tissent ces liens de dépendances et d'interdépendances entre acteurs de la formation.

13. La propriété d'affordance est issue de l'approche écologique de la perception (Gibson 1979). Le terme anglais n'a pas d'équivalent exact en français. L'affordance indique qu'un artefact, par son aspect perceptible, incite l'utilisateur à effectuer les actions que permet cet artefact. Par exemple, un bouton suggère que l'on peut appuyer dessus. Norman propose l'exemple célèbre des poignées de portes dont la disposition et la forme inciteront plutôt à tirer, pousser ou tourner (Norman 1988). 


\section{Phase d'implication, d'engagement et d'identification}

L'implication d'un sujet dans une démarche d'apprentissage, l'expression, le maintien et le renforcement de sa motivation intrinsèque sont influencés par le contexte dans lequel se déroule l'action. Ce contexte est plus précisément défini par, d'une part la valeur que le sujet donne à l'activité proposée, d'autre part, en fonction de son sentiment de compétence vis-à-vis de la tâche à réaliser, et enfin, du degré de liberté qui lui est offert dans le choix de ses activités. Pour ce faire, le tuteur agit dans l'identification et la reconnaissance du projet de l'apprenant. L'objectif de cette première phase est d'initier une relation intersubjective et réciproque entre les acteurs engagés dans la réalisation du projet d'apprentissage de l'apprenant. Cette phase est celle de l'engagement dans la démarche, de nature motivationnelle. L'établissement de cette relation intersubjective semble être nécessaire pour harmoniser le projet de l'apprenant et l'offre institutionnelle, et développer ainsi la persévérance de l'apprenant.

\section{Phase de distanciation et compréhension}

L'accompagnement est pensé comme une démarche révélatrice d'un sens caché et d'intégration de ces informations dans une dynamique de modifications des schèmes du sujet. Il est un acte formatif codirigé par le sujet et l'accompagnateur. Ceci rejoint le modèle dialogique du coinvestissement développé par Pineau et Legrand (1993). Pour ces auteurs «l'explicitation du savoir implicite est une ouvre conjointe, nécessitant un coinvestissement des acteurs impliqués dans les deux opérations d'énonciation et de travail sur l'énoncé. » C'est par l'interprétation conjointe des deux protagonistes de la relation que se construit le sens, chacun agissant sur une partie de la pièce du puzzle et sur ses relations avec les autres pièces. La phase de distanciation est celle au cours de laquelle le sujet se remémore, se souvient et saisit le sens des informations produites, et est capable d'exprimer avec ses propres mots ce qu'il sait. La distanciation est également l'étape d'appropriation des difficultés rencontrées, de leur reconnaissance et de leur acceptation.

\section{Phase d'intégration et de transformation}

Mais il semble que cette boucle comprenne un troisième élément, l'intégration qui concrétise la transformation du système initial et qui doit notamment permettre de définir un état d'équilibre du système que représente le dispositif. Cette phase de transformation se déroule dans cette zone proximale d'actualisation dont les limites peuvent se déplacer au fur et à mesure des différents cycles de cette spirale. La transformation est une forme d'étape créative. A partir de l'analyse qu'il porte sur l'information produite lors de la phase précédente, l'individu est en capacité d'accepter des adaptations et d'établir de nouvelles relations entre les différents acteurs du dispositif. La transformation est l'étape au terme de laquelle, après avoir 
reconnu les difficultés, les acquis, le sujet se reconnaît comme pouvant vivre dans une nouvelle organisation.

Cette approche circulaire est plutôt spiralée et progressive et fait se croiser deux dialectiques : l'expérience et l'existence d'une part, et d'autre part la réflexion et la conceptualisation. Antérieurement, Kolb a proposé quatre étapes structurantes de la construction des savoirs :

1. l'explicitation de l'expérience concrète dans laquelle l'individu s'est impliqué ;

2. l'observation réflexive sur la signification de l'expérience pour l'individu ;

3. la conceptualisation abstraite pour cerner les idées hypothétiques sousjacentes ;

4. l'expérimentation active pour vérifier ces idées dans de nouvelles situations.

Les étapes 1 et 2 de ce modèle structureraient ce que nous nommons « distanciation/compréhension ». En effet, c'est bien dans cette phase que le sujet produit de manière distanciée et accompagnée un sens à son vécu. Les expressions émotionnelles et discursives sont productrices d'informations sur le sens de l'expérience. Les étapes 3 et 4 proposées par Kolb (1984) sont intégrées à la troisième phase de notre proposition qui, au-delà de ce que propose cet auteur, vise la transformation du sujet via la réalisation d'apprentissages et l'actualisation du dispositif.

Pour comprendre cette dynamique de coconstruction et les relations entre les différents acteurs, nous considérons ce processus comme un acte de transformation par lequel se construit l'efficacité (Jullien, 1996). Dans cette perspective, le tutorat, au nom de la nécessaire réciprocité éducative indispensable et structurante, ne peut être uniquement fondé sur la maîtrise d'un savoir ou la maîtrise d'outils méthodologiques, mais également sur des capacités humaines dont Rogers (1974) nous à dressé les principales caractéristiques.

Coconstruire suppose l'acceptation de l'autre dans sa diversité dans ce qu'il n'est pas et que nous souhaiterions qu'il soit ${ }^{14}$, c'est également accepter de lâcher prise et par conséquent que l'institution autorise des degrés de liberté d'action et de pensée. Cette posture suppose que le tuteur soit en capacité d'établir une relation d'aide étayante et contenante comme la définit Rogers : empathie, considération positive inconditionnelle, congruence. Cette analyse permet de voir en quoi l'accompagnement peut être un acte qui est à la fois partage et déplacement comme le définit Abels (1998) : "L'accompagnement a un sens de partage, d'être avec, à côté de et non à la place; chacun est considéré comme sujet et acteur, aucun n'est

14. Lors d'un entretien, un tuteur relata un cas d'incompréhension consécutif à une représentation erronée de sa part de l'apprenant. Répondant à une question d'un apprenant, il construisit sa réponse en pensant que ce dernier pensait telle chose jusqu'à ce qu'il comprenne et accepte que l'apprenant qu'il se représentait n'était pas celui qui le questionnait. 
l'objet de l'autre. \" Rien n'est jamais acquis, ni modèle, ni résultat. L'industrialisation définie en back-office, propose des ressources et procédures préconstruites dont l'actualisation se réalise de manière singulière, participative dans le front-office. Cette actualisation s'inscrit dans une dynamique collaborative en cela qu'il y a construction d'une signification commune (Littleton et Häkkinen, 1999). C'est un processus de création partagée : «deux individus ou plusieurs possédant des compétences complémentaires interagissent pour créer une compréhension partagée qu'aucun ne possédait auparavant ou qu'il n'aurait pu construire seul». (Schrage, 1991). Cette dimension collaborative suppose la reconnaissance de l'autre. Pour Lewis (2003), le «potentiel collectif ne peut se réaliser qu'à la condition que chaque membre de la communauté soit conscient du savoir des autres et capable de capitaliser cette expérience dans l'échange - en offrant et en recevant de l'aide avec les autres ». L'acceptation de l'autre dans sa considération inconditionnelle par le tuteur devient une condition de l'exercice tutoral pour que se réalise cette collaboration. Tuteurs, apprenants, participent conjointement à la réalisation de l'apprentissage, objet reconnu comme bien commun lors de la phase d'implication. Cette relation réciproque, lien social éducatif, suppose une conscience et une mémoire commune et requiert une organisation explicite de la capitalisation des traces des échanges. Ces informations trouvent une double utilité : rétroactive et projective. Elles permettent d'une part de capitaliser les expériences antérieures et contribuent d'autre part à la planification de la poursuite du projet d'apprentissage.

\section{Tutorat : exercice d'une fonction contenante pour l'actualisation}

L'actualisation du dispositif, exposée dans sa fonction et sa dynamique, suppose un accompagnement spécifique des apprenants par les tuteurs dont l'objectif est l'initialisation d'une fonction contenante transformatrice.

Mialaret (1991) définit l'éducation comme un espace-temps à vivre, à partager, à habiter ensemble, pour une action réciproque de deux sujets l'un vers l'autre qui aboutirait à la transformation des deux personnalités mises en présence. Dans un contexte conventionnel, d'éducation formelle, l'unité de temps, de lieu et d'action structurent la formation, laissant plus ou moins d'initiative à l'apprenant, véritable dynamique spatio-temporelle mobilisatrice de différentes ressources : le formateur, le groupe, l'environnement. Cet espace-temps social de l'interaction éducative, construit une dynamique relationnelle d'étayage puis de désétayage nécessaire à la construction par l'apprenant de ses propres apprentissages. Les approches cognitives et interactionnistes de la formation et de l'apprentissage ont fortement développé le concept d'interaction de tutelle, qui précise l'importance de différents aspects de guidance dans l'acte éducatif. Concept développé par Bruner, l'étayage est un dispositif d'aide et de soutien approprié. Le tuteur peut aider l'apprenant à dépasser ses découvertes spontanées et faire «un pas de plus » vers la prise de conscience, facteur décisif de l'apprentissage (Raynal et Rieuner, 1997). L'étayage assure une guidance qui est relayée par le désétayage, qui prive peu à peu le sujet de l'aide dont 
il disposait. C'est par le désétayage que l'on permet au sujet d'accéder seul à la maîtrise de la compétence: niveau d'accommodation et d'équilibration (Piaget, 1970). Cet espace-temps social de la formation définit une limite, cette thèque, enveloppe contenante, qui différencie le dedans et le dehors identifiant le cadre d'action, celui de l'exercice de la liberté de choix. Physique et symbolique, ce cadre résulte d'une coconstruction entre l'accompagnateur et l'apprenant. Le cadre sans être une structure fermée qui définit et autorise une multiplicité d'actions, d'expression, d'échanges internes au dispositif prescrit et externes, qui organisent l'actualisation du dispositif.

Mobiliser la notion de cadre dans un contexte de formation ouverte pourrait se révéler être un non-sens, une gageure, voire une injonction paradoxale qui place le sujet dans cette double contrainte de l'initiative dans un espace clos. L'hypothèse directrice de ce propos postule l'exercice d'une fonction contenante de la dynamique d'actualisation des fonctionnalités du dispositif par l'apprenant. Expliciter cette notion de cadre revient à préciser ses différentes fonctions : fonction d'expression, fonction contenante, fonction étayante, fonction transitionnelle. Le cadre a pour fonction de potentialiser les capacités de changement du sujet, et par conséquent d'apprentissage.

La fonction du cadre est d'accueillir les besoins, les sollicitations et demandes de l'apprenant. Le cadre contient autant qu'il gère la transformation du dispositif. De même que la classe constitue à la fois un espace clos et une porte ouverte sur le monde, le dispositif de formation ouverte et à distance joue cette double fonction : celle de la protection et de la découverte. Par sa fonction d'espace protégé (Bourgeois, 1996) et étayé, il permet au sujet de transformer les informations issues de la phase de distanciation d'en tester la nouvelle validité en limitant les risques d'entropie. Le cadre devient l'espace de « jeu » du « je ». C'est un espace de jeu au sein duquel le sujet peut déposer ses attendus, ses difficultés, les revivre, en comprendre le sens et bâtir un nouveau cheminement, une nouvelle organisation qui trouve ici l'occasion d'être expérimentée.

Si le cadre est un espace de jeu, il définit des règles du jeu. Au plan communicationnel cela se traduit par des niveaux informationnels acceptables et pertinents. Il revient alors au tuteur d'assurer cette gestion, dans une dualité qui résulte de ce que le sujet exprime et de ce que l'accompagnateur peut traiter. Ce dernier doit alors se situer à un double niveau, celui du contenant et celui de l'interprétation, double activité qui sans cesse doit être dirigée vers le projet d'autrui, posant ainsi la question de la limite des compétences et de la nécessité de relais. L'accompagnateur/tuteur doit être capable de gérer des capacités interprétatives et intégratives.

Initialiser un processus formatif revient dans un premier temps à établir une communication contenue qui actualise les potentialités communicatives du sujet pour l'aider à sortir de la simple quête du connu et des habitus pour tendre vers un processus créatif efficient. La fonction du cadre est de contenir les projections du 
sujet et de l'autoriser à dire l'indicible pour lui permettre de produire du sens à ses dysfonctionnements, aux difficultés et obstacles qu'ils rencontrent. Donner au cadre, au contenant une fonction de conteneur c'est lui reconnaître une fonction toute particulière de médiation, de système par lequel s'expriment pulsions et discours. La finalité de la communication entre tuteurs et apprenants dépasse la simple validation et évaluation des activités d'apprentissage pour tendre vers une réciprocité qui organise la coconstruction d'un dispositif opérant pour l'ensemble des acteurs.

L'une des fonctions du cadre est d'assurer un étayage multiple. Notion convoquée par Kaës (1997), elle indique explicitement que l'étayage ne peut être réalisé selon une seule dimension du sujet, mais doit bien prendre en compte ses différentes composantes. Ainsi l'étayage ne saurait être uniquement affectif ou cognitif, il doit être l'un et l'autre en fonction de la dynamique d'évolution du sujet. Nous proposons que l'accompagnateur exerce sa capacité à agir selon différents plans et pour différents objectifs. Ainsi il peut agir au plan de l'étayage cognitif tout en agissant conjointement au niveau affectif. Son savoir-faire s'exerce dans cette complémentarité d'action. La fonction du cadre dépasse dès lors sa simple fonction de contenant pour devenir conteneur, c'est-à-dire un espace transitionnel interprétatif, espace communicationnel et étayé de transformation du sujet.

Espace potentialisateur, le cadre assure la transition entre un état $n$ et un état $n+1$ du sujet et par conséquent de son rapport au dispositif. Cette fonction transitionnelle a été largement développée par Winnicott (1975) qui conçoit la transition comme un espace de création accompagnée : «L transition winnicottienne est conçue comme un dispositif intermédiaire, qui répond à l'expérience de la perte de l'objet par des satisfactions substitutives provisoires, étayées et confirmées par l'adulte, jusqu'à ce que soient constituées des capacités de représentation de l'objet plus complètes, quasi opératoires. "

L'une des fonctions de cet espace serait la recherche de l'intentionnalité, de la production de sens, construction intersubjective entre apprenants et tuteurs. Ces dispositifs organisent les actes par lesquels l'accompagnateur/tuteur et l'apprenant agissent de manière à la fois réciproque et asymétrique pour la reconnaissance du sujet. C'est par et dans cet espace potentiel que le sujet renaît et se re-connaît comme un sujet en développement et accepte son engagement dans l'acte formatif. L'accompagnateur/tuteur est initiateur de cette verbalisation, forme de conscientisation, tout en gérant cette initialisation dans ce qu'il perçoit comme étant l'espace d'acceptabilité par le sujet de cette prise de conscience. Double niveau d'intervention pour l'accompagnateur : dans la relation, et au-dessus de la relation. Il est bien dans les différentes postures définies par Le Bouëdec (1998), à la fois directeur, guide et accompagnateur. 
Si la formation est le déplacement dans l'espace problème ${ }^{15}$ du sujet, c'est également un déplacement des acteurs dans un espace symbolique et relationnel. Cet espace d'action, de mise en relation de l'un et l'autre permet à chacun de se déplacer de manière optimale afin de trouver le meilleur angle de vue, d'analyse du problème. Pour Pineau et Legrand (1993) «L'espace est occupé par un déplacement réciproque qui cherche le point de vision optimum permettant au locuteur de se distancier de sa vie en s'approchant des systèmes de compréhension, et à l'interlocuteur de s'approcher suffisamment de cette vie en sortant de ses systèmes conceptuels. » Cet espace, créé par les déplacements des acteurs, du sujet et de l'accompagnateur, constitue l'espace communicationnel d'investigation, d'interprétation et de transformation de l'espace problème. C'est l'espace transitionnel physique et symbolique au sein duquel, le sujet se trans-forme, se construit une forme nouvelle par recomposition de ces aires du soi et de leurs interactions. Ainsi l'actualisation est inscrite dans une double transformation, celle des acteurs et celle du dispositif.

\section{Conclusion...}

Le tutorat, habituellement pensé et réfléchi comme l'accompagnement de la réalisation des apprentissages est ici analysé dans sa fonction anticipatrice et régulatrice de l'actualisation des potentialités d'un dispositif prescrit. Les différents travaux exposés dans cet article donnent à penser que le pouvoir d'agir, de décider, d'intervenir, que chacun reconnaît à l'autre, participerait à l'actualisation du dispositif prescrit en un dispositif vécu. Cette actualisation se traduit par une reconfiguration du dispositif initial. Acte de trans-formation par lequel apprenants et tuteurs s'engagent activement pour définir la configuration la plus propice au regard du contexte et des capacités de l'apprenant pour contenir et soutenir l'apprentissage. Le dispositif ne peut être réduit à un objet industriel figé, mais considéré comme un système ouvert dont les composantes et relations prennent sens dans une dynamique de sémiogénèse partagée entre les différents acteurs engagés dans l'acte formatif. Les actes de communication et d'information qui organisent l'apprentissage dans de tels dispositifs sont contextuellement situés. Ce processus de reconnaissance de l'objet (artefact) par le sujet, d'un sujet par un autre sujet, contribue à l'appropriation ou réappropriation ${ }^{16}$ de son pouvoir d'action par l'apprenant. Cette coconstruction semble orientée par la quête d'une intentionnalité partagée dirigée par la reconnaissance mutuelle d'un bien commun pour partie de nature symbolique où le partage de signifiants participe à cet imaginaire commun. Les habitus et représentations que les acteurs mobilisent dans une telle dynamique semblent

15. Cette notion d'espace problème peut être définie, à l'instar de Richard, comme l'espace de recherche qui correspond à l'interprétation que s'est donné le sujet d'une situation (Richard, 1995).

16. Nous sommes ici proche de l'une des définitions données de l'empowerment. 
orienter la dynamique d'actualisation du potentiel proposé par le dispositif prescrit. Le tutorat devient un agir en situation, pour l'exercice duquel, il ne suffit plus seulement être capable d'exécuter le prescrit, mais d'être en capacité d'aller au-delà $\mathrm{du}$ prescrit (Le Boterf, 2002) pour coconstruire une forme actualisée de l'accompagnement de la réalisation d'un projet d'apprentissage explicite et explicité. Cette première esquisse suppose de multiples approfondissements et tout particulièrement l'analyse des dynamiques spatio-temporelles et sociales à l'œuvre dans ce processus dual d'actualisation.

\section{Bibliographie}

Abels C., «L'accompagnement de l'enfant placé dans son travail d'histoire de vie », Pineau (eds), Accompagnements et histoire de vie, Paris, L'Harmattan, 1998.

Albertini J.-M., «Une nouvelle économie de la formation?», Etudes et expérimentations en formation continue, $\mathrm{n}^{\circ} 16$. juillet-octobre 1992, p. 3-8.

Beguin P., Cerf, M., Formes et enjeux de l'analyse de l'activité pour la conception des systèmes de travail, http://www.activites.org, consulté le 23 juin 2004.

Bourgeois E., «Identité et apprentissage », Education Permanente, vol. 1996-3, n 128, 1996, p. 27-35.

Daniellou F., «Ergonomie et conduite de projet», Falzon P., L'ergonomie, (coord), Paris, PUF, 2004.

Docq F., Daele A., «De l'outil à l'instrument : des usages en émergence », Charlier B., Peraya D., (eds), Technologie et innovation pédagogique, Bruxelles, De Boeck, 2003, p. 113-128.

Eisen A., «Survey of neighborhood-based, comprehensive community empowerment initiatives », Health Education Quaterly, vol. 21, n² 2, 1994, p. 235-252.

Engeström Y., Learning by expanding : an activity-theoretical approach to developmental research, Helsinki, Orienta-Konsultit, 1987.

Fastrez P., «Hypertextualité et modification du rapport au savoir », Education Permanente, vol. 2002-3, $\mathrm{n}^{\circ} 152,2002$, p. 31-42.

Giordan A., De Vecchi G., Les origines du savoir, Neuchatel, Delachaux \& Niestlé, 1987.

Glikman V., «Apprenants et tuteurs : une approche européenne des médiations humaines », Education Permanente, vol. 2002-3, n 152, 2002, p. 55-69.

Henderson A., Kyng M., "There is no place like home. Continuing design in use ", Greenbaum J., Kyng M. (Eds.), Design at work: cooperative design of computer systems, Hillsdale, Lawrence Erlbaum Associates Publishers, 1991.

Houssaye J., Le triangle pédagogique, Paris, Peter Lang, 1988.

Jullien F., Traité de l'efficacité, Paris, Grasset, 1996. 
Kolb D., Experiential Learning, Eaglewood Cliffs, Prentice Hall, 1984.

Kaës R., Crise, Rupture et dépassement, Paris, Dunod, 1997.

Kuutti K., "The concept of activity as a base unit of analysis for CSCW research", Proceedings of Second Conference on CSCW, Amsterdam, Kluwer Academic Publisher, 1991, p. 249-264.

Leboterf G., Ingénierie et évaluation des compétences, Paris, éd d'Organisation, 2002.

Le Bouëdec G., « Diriger, suivre, accompagner : au-dessus, derrière, à côté », Cahiers BinetSimon, $\mathrm{n}^{\circ} 655,1998$, p. 53-63.

Leontiev A.N., Activité, Conscience, Personnalité, Moscou, Editions du Progrès, 1984.

Lewis R., « Pourquoi apprendre à collaborer », Charlier, B., Peraya, D., (eds), Technologie et innovation pédagogique, Bruxelles, De Boeck, 2003, p. 137-139.

Linard M., « Nouvelles technologies et formation », Eduquer et former, Sciences Humaines, Hors Série, n 12, Auxerre, 1996, p. 325-330.

Littleton K., Häkkinen P., «Learning together: understanding the processes of computerbased collaborative learning », P. Dillenbourg (ed), Collaborative learning, cognitive and computational approaches, London, Elsevier Science, 1999, p. 21.

Mialaret G., Pédagogie générale. Paris, PUF, 1991.

Moore M.G., Kearsley G., Distance Education - A System View, Belmont (USA), Wadsworth Publ., 1996.

Ochanine D., «Le rôle des images opératives dans la régulation des activités de travail », Psychologie et Education, vol. 2, n 3, 1978, p. 63-79.

Paquelin D., Choplin H., Projet MIReHD-EFAd, Rapport d'Evaluation, GET, 2002.

Paquelin D., «Accompagnements, émergence de la forme », Colloque L'Accompagnement et ses paradoxes, Fontevraud, (22-23-24 mai), 2003, cédérom.

Paquelin D., Choplin H., « Du prescrit au vécu, l'enjeu des régulations », B. Albero, (sld), Autoformation et enseignement supérieur, Paris, Hermes-Science, 2003, p. 167-183.

Paquette G., L'ingénierie pédagogique, Québec, PUQ, 2002.

Pastré P., «Comprendre après coup grâce à la simulation », Education Permanente, vol. 1999-2, n 139, 1999, p. 90-115.

Pineau G., Legrand J.-L., Les histoires de vie, Paris, PUF, 1993.

Pineau G., Dictionnaire Encyclopédique de l'Education et de la Formation, Paris, Nathan Université, 2000.

Rabardel P., Les hommes et les technologies: approches cognitives des instruments contemporains, Paris, A. Colin, 1995.

Raynal F., Rieuner A., Pédagogie, dictionnaire de concepts clés, Paris, ESF, 1997. 
$182 \mathrm{D} \& \mathrm{~S}-2-3 / 2004$. Enigmes de la relation pédagogique

Richard J.-F., Les activités mentales, Paris, Armand Colin, 1995.

Rogers C. R., In restrospect 46 years, American Psychology, vol. 29, n 2, p. 116.

Samurçay R., Pastré P., «La conceptualisation des situations de travail dans la formation des compétences », Education Permanente, vol. 2, n 123, 1995, p. 13-31.

Schrage S., Shared minds: the new technologies of collaboration, New York, Prentice Hall, 1991.

Suchman L., Plans and situated actions, Cambridge, University Press, 1987.

Vicente K.-J., Cognitive work analysis: toward safe productive and healthy computer-based work, Londres, Lawrence Erlbaum Associate Publishers, 1999.

Vygostsky L., Mind in society: the development of higher psychological processes, Cambridge, Harvard Press University, 1978.

Weill-Fassina A., Rabardel P., Dubois D., Représentation pour l'action, Toulouse, Octarès, 1993.

Winnicott D., Jeu et Réalité, Paris, Gallimard, 1975. 